\title{
Ortorexia nervosa em estudantes de nutrição: associações com o estado nutricional, satisfação corporal e período cursado
}

\author{
Orthorexia nervosa in nutrition students: association with \\ nutritional status, body satisfaction and coursed period \\ Fernanda R. O. Penaforte, ${ }^{1,2,3}$, Sabrina M. Barros0 3,4, Maria Eduarda Araújo1, Camila C. Japur2,5
}

\section{RESUMO}

Objetivo: Identificar a prevalência de comportamentos com tendência a ortorexia nervosa (ON) e suas associações com o estado nutricional, satisfação corporal e período cursado em estudantes de nutrição. Métodos: Participaram do estudo 141 estudantes (com idade média de 21,5 $\pm 3,5$ anos). A tendência à ON foi avaliada por meio do questionário para identificação da ON (ORTO-15) e a satisfação corporal, pelo Body Shape Questionnaire. A classificação do estado nutricional foi feita pelo IMC $\left(\mathrm{kg} \cdot \mathrm{m}^{-2}\right)$. Associações entre as variáveis foram avaliadas pelo teste do qui-quadrado $(p<0,05)$. Resultados: Comportamentos alimentares com tendência a ON foram identificados em 87,2\% dos estudantes. Os estudantes com ON apresentaram mais insatisfação corporal $\left(X^{2}=7,48 ; p=0,005\right)$ e excesso de peso $\left(x^{2}=6,56 ; p=0,010\right)$. Não foi observada associação entre a ON e o período cursado. Conclusão: Os resultados demons-

\section{Palavras-chave \\ Ortorexia nervosa, comportamento alimentar, imagem corporal, estado nutricional.} tram que os estudantes de nutrição parecem ser uma população especialmente em risco para tendência à $\mathrm{ON}$, independentemente do período cursado. A maior frequência de estudantes com ON e excesso de peso e insatisfação corporal pode sugerir que tais condições, que comumente cursam com preocupações com alimentação saudável, podem facilitar o início do desenvolvimento de comportamentos alimentares associados a ON.

\section{ABSTRACT}

Objective: To identify the prevalence of orthorexia nervosa (ON)'s risky behaviors and their association with nutritional status, body satisfaction and coursed period in nutrition students. Methods: The study included 141 students (mean age $21.5 \pm 3.5$ years). The risk behavior to ON was assessed by using the questionnaire to identify ON (ORTHO-15) and body satisfaction with the Body Shape Questionnaire. The classification of nutritional status was evaluated by BMI $\left(\mathrm{kg} \cdot \mathrm{m}^{-2}\right)$. Association between variables was evaluated by chi-square test $(p<0.05)$. Results: Eating behaviors at risk for ON were identified in $87.2 \%$ of students. Students with ON had more body dissatisfaction $\left(X^{2}=7.48 ; p=0.005\right)$ and overweight $\left(X^{2}=6.56 ; p=0.010\right)$. No association was observed between the $\mathrm{ON}$ and coursed period. Conclusion: The results show that Nutrition students seem to be a population at a particular risk for ON, regardless

\footnotetext{
1 Universidade Federal do Triângulo Mineiro (UFTM), Departamento de Nutrição.

2 Laboratório de Práticas e Comportamento Alimentar (PratiCA).

3 Universidade Federal do Triângulo Mineiro (UFTM), Núcleo de Avaliação Psicológica e Investigação em Saúde (NAPIS), Programa de Pós- Graduação em Psicologia.

4 Universidade Federal do Triângulo Mineiro (UFTM), Departamento de Psicologia.

5 Universidade Federal de Uberlândia (UFU), Curso de Nutrição.
} 


\section{Keywords}

Orthorexia nervosa, feeding

behaviour, body image,

nutritional status of the coursed period studied. The higher frequency of students with ON and overweight and body dissatisfaction may suggest that such conditions, which commonly occur with concerns about healthy eating, may facilitate the initiation of the development of eating behaviors associated with ON.

\section{INTRODUÇÃO}

A ortorexia nervosa $(\mathrm{ON})$ vem sendo apresentada na literatura como um novo tipo de desvio do comportamento alimentar, do tipo obsessivo-patológico, no sentido de preocupação excessiva com a qualidade dos alimentos e pureza da dieta'. O termo "ortorexia" significa "alimentação correta" e é derivado do grego "orto", que significa "correto, direito", e "rexia", que corresponde a apetite ${ }^{1,2}$.

A ON foi inicialmente descrita pelo médico americano Steven Bratman, em 1997, como uma fixação pela saúde alimentar caracterizada por obsessão doentia com o alimento biologicamente puro, acarretando restrições alimentares importantes ${ }^{3,4}$. Os indivíduos com ON apresentam preocupação exagerada com a qualidade dos alimentos, sendo essa sua principal preocupação, restringindo ao máximo o consumo dos alimentos que contenham substâncias como corantes, conservantes, pesticidas, ingredientes transgênicos, gorduras menos saudáveis e excesso de sal e açúcar. Eles tendem, também, a excluir de sua alimentação alimentos que não sejam orgânicos e se preocupam excessivamente com o seu modo de preparo e origem da matéria-prima ${ }^{1,2}$. A quantidade de alimentos consumida e a perda de peso não são consideradas prioridades para os sujeitos com ON ${ }^{5}$, muito embora a perda de peso seja apontada como um possível fator desencadeador desse desvio do comportamento alimentar ${ }^{6,7}$.

Estar atento ao que se come e preocupar-se em ter uma alimentação saudável é uma atitude, em geral, benéfica para a saúde e para a qualidade de vida. Isso pode fazer com que a ON fique camuflada em um falso "discurso de saúde", impedindo que seja encarada como um problema, tendo em vista a dificuldade em entender e aceitar que comportamentos "saudáveis" podem levar a danos para a saúde. Porém, é importante destacar que, quando a preocupação com o comer saudável é tamanha que se aproxima de uma obsessão, a vida do indivíduo acaba girando em torno da comida, o que pode acarretar grandes restrições alimentares e complicações sociais².

O primeiro instrumento validado com objetivo de identificar indivíduos com sintomas de ON foi publicado por Donini et al. em 20057, e apenas em 2012 Pontes publicou a versão traduzida e adaptada culturalmente do ORTO-15 para o Brasil| ${ }^{8}$. Mais recentemente, Dunn e Bratman propuseram alguns critérios para o diagnóstico da ON. De acordo com essa proposta, o indivíduo com ON deve apresentar as seguintes características: A1) foco excessivo no comer saudável; A2) respostas físicas e emocionais exageradas às transgressões dietéticas, que diferenciam um desejo normal de se ter uma alimentação saudável de uma potencial doença; A3) restrições alimentares que aumentam progressivamente ao longo do tempo, podendo abarcar a exclusão de grupos alimentares inteiros; e um dos seguintes prejuízos físicos e/ ou emocionais/psicológicos, decorrentes da preocupação excessiva com a alimentação saudável: B1) desnutrição, B2) angústia ou prejuízos sociais, acadêmicos ou vocacionais e B3) imagem corporal positiva, autoestima e satisfação pessoal excessivamente dependentes da adesão à dieta saudável' . Todavia, a ON ainda não é reconhecida como transtorno alimentar pelo Diagnostic and Statistical Manual of Mental Disorders $5(\mathrm{DSM}-5)^{10}$.

Apesar de ainda serem escassos, principalmente se considerarmos a complexidade e a relevância do tema, estudos feitos com diferentes públicos encontraram prevalências que variaram entre $53,7 \%$ em estudantes poloneses do ensino médio ${ }^{11}, 68,6 \%$ em estudantes universitárias polonesas do sexo feminino ${ }^{12}, 71,2 \%$ em universitários americanos ${ }^{13}, 86,0 \%$ em membros de uma comunidade de Ashtanga Yoga, na Espanha ${ }^{14}$, e em 45,5\% em médicos residentes da Turquia ${ }^{15}$. Avaliando a prevalência em uma amostra com características ocupacionais mais diversificada, Donini et al. encontraram prevalência de 6,9\% ${ }^{16}$.

Evidências na temática têm apontado que profissionais de saúde em geral, em especial os nutricionistas e estudantes de nutrição, são mais suscetíveis a apesentarem comportamentos alimentares compatíveis com a ON. Pela necessidade de lidarem diretamente com os temas saúde e/ou alimentação saudável ${ }^{17-22}$, os nutricionistas e os estudantes de nutrição são pressionados a terem uma alimentação adequada e saudável, servindo como uma espécie de "modelo alimentar" para a sociedade. Nesse caso, comer corretamente vai além dos princípios de promover saúde e acaba sendo um comportamento socialmente aceitável e desejável, colocando esse grupo em situação de vulnerabilidade para o desenvolvimento da ON ${ }^{1,8}$. Outro reflexo desse fenômeno recai sobre o possível crescimento dessa vulnerabilidade durante o curso de graduação em nutrição, uma vez que conhecimentos específicos sobre composição de alimentos, dietas e relação entre alimentos e saúde vão sendo ampliados e aprofundados ao longo da formação.

Por se tratar de um tema relativamente novo na área da saúde, são escassos os trabalhos, notadamente no cenário 
nacional, que exploraram o tema da ON. Os estudos nacionais disponíveis se detiveram, prioritariamente, em fazer reflexões teóricas sobre a temática ou em verificar a prevalência de ON 18,18,19,23, mas são poucos os que buscaram explorar associações entre esse desvio do comportamento e variáveis como período cursado ou satisfação corporal, que são fatores que podem estar relacionados com a ON. Diante desse panorama, o objetivo do presente estudo foi identificar a prevalência de comportamentos alimentares com tendência à ON entre estudantes de nutrição de uma universidade do interior de Minas Gerais, bem como verificar suas associações com o estado nutricional, satisfação corporal e período cursado.

\section{MÉTODOS}

\section{Desenho do estudo e sujeitos da pesquisa}

Trata-se de um estudo de corte transversal e de caráter exploratório e correlacional. O estudo foi realizado com estudantes de graduação do curso de Nutrição da Universidade Federal do Triângulo Mineiro (UFTM). Foram incluídos no trabalho estudantes de ambos os sexos, com idade igual ou superior a 18 anos, regularmente matriculados em qualquer período do curso, e excluídos aqueles que se negaram a realizar a avaliação antropométrica, que não preencheram corretamente todos os questionários do estudo e que apresentavam diagnóstico autorrelatado de diabetes mellitus, hipertensão arterial sistêmica ou doença cardiovascular, pois envolvem orientações nutricionais específicas que poderiam gerar viés na avaliação da ON.

Para o cálculo amostral, considerou-se o total de estudantes matriculados no curso de Nutrição no ano de 2014 (218), prevalência desconhecida (50\%), erro tolerável de 5\% e intervalo de confiança de $95 \%$, totalizando uma amostra de 140 estudantes.

O estudo foi aprovado pelo Comitê de Ética em Pesquisa (CEP) da UFTM (protocolo no 631.104) e todos os participantes assinaram o Termo de Consentimento Livre e Esclarecido (TCLE).

\section{Instrumentos}

a) Avaliação da Ortorexia Nervosa: para essa avaliação, foi utilizado o questionário para identificação da ON - ORTO-15 -, em sua versão traduzida e adaptada culturalmente para o português por Pontes ${ }^{8}$. O ORTO-15 é um questionário autoaplicável, composto por 15 questões que avaliam atitudes em relação a seleção, aquisição, preparo e consumo de alimentos. Cada questão apresenta quatro opções de resposta (sempre, muitas vezes, algumas vezes, nunca). A pontuação total do questionário é de, no mínimo, 15 e, no máximo, 60 pontos. Pontuações abaixo de
40 pontos sinalizam comportamentos com tendência à ON?

b) Avaliação da satisfação corporal: a satisfação corporal foi avaliada pelo Body Shape Questionnaire (BSQ), na versão validada para o português ${ }^{24}$. Trata-se de questionário autoaplicável, composto por 34 perguntas, originalmente desenvolvido e validado para mulheres. Portanto, no presente estudo, a avaliação da satisfação corporal foi realizada apenas nos participantes do sexo feminino. As respostas são apresentadas em escala tipo likert de 6 pontos, indo de 1 (nunca) a 6 (sempre). Pontuações menores que 80 sinalizam ausência de insatisfação com a imagem corporal; entre 80 e 110, insatisfação leve; entre 111 e 140, insatisfação moderada; e maior que 140, insatisfação grave ${ }^{25}$.

c) Avaliação antropométrica: o peso (kg) e a altura (m), para cálculo do índice de massa corporal (IMC - kg/m²), foram aferidos de acordo com técnicas padronizadas $^{26}$. A classificação do estado nutricional pelo IMC foi feita de acordo com os pontos de corte propostos pela Organização Mundial da Saúde $(\mathrm{OMS})^{27}$, para aqueles com idade superior a 18 anos, e pela utilização das curvas de IMC para sexo e idade, para aqueles com idade igual a 18 anos $^{28}$.

\section{Procedimentos}

O convite para participação no estudo foi feito em sala de aula, após anuência do professor responsável pela aula, e o processo de coleta de dados ocorreu em duas etapas. Na primeira etapa, após os esclarecimentos acerca dos objetivos e procedimentos concernentes ao estudo, os estudantes que concordaram em participar assinaram o TCLE e preencheram os questionários autoaplicáveis em sala de aula, na seguinte ordem: (1) ORTO-15 e (2) BSQ. Em seguida, foram feitos os agendamentos individuais e orientações para a realização da avaliação antropométrica, que ocorreram em até sete dias após a realização da primeira etapa.

\section{Análise de dados}

Realizaram-se análises de frequência absoluta e relativa, tendência central (média) e dispersão (desvio-padrão). Em seguida testou-se a normalidade dos dados pelo teste de Kolmogorov-Smirnov. Como os dados não apresentaram distribuição normal ( $K S=0,108 ; p=0,001)$, as associações entre as variáveis foram avaliadas pelo teste do qui-quadrado e as correlações pelo teste de Spearman. Para verificar fatores independentemente associados à $\mathrm{ON}$, realizou-se análise de regressão logística considerando como variável independente a presença/ausência de ortorexia e como variáveis independentes: sexo, idade dicotomizada pela média, período dicotomizado, altura, peso, IMC (considerando a covariância entre essas medidas), insatisfação corporal e excesso de peso. O nível de significância adotado foi de 5\%. 


\section{RESULTADOS}

\section{Caracterização dos sujeitos}

Os estudantes tinham idade média de 21,5 anos ( $D P \pm 3,5$ ), sendo $90,8 \%$ do sexo feminino. O IMC médio dos sujeitos avaliados foi de $22,9 \mathrm{~kg} / \mathrm{m}^{2}(\mathrm{DP} \pm 4,1)$ e, de acordo com a classificação do estado nutricional, a maior parte deles $(75,9 \%)$ encontrava-se eutrófica e $24,1 \%$ apresentavam excesso de peso, sendo 19,1\% com sobrepeso e 5,0\% com obesidade.

\section{Ortorexia nervosa e satisfação corporal}

Entre os avaliados, 87,0\% apresentaram comportamento alimentar com tendência à ON. Em relação à satisfação corporal, 57,8\% dos estudantes apresentaram algum grau de insatisfação corporal (variando de leve a grave).

Entre as estudantes, foi observada associação significativa entre ON e insatisfação corporal ( $r h o=0,25 ; p=0,001$ ), com maior frequência de insatisfação corporal entre as estudantes com comportamento alimentar com tendência para a ON $\left(x^{2}=7,48 ; p=0,005\right)$ - Tabela 1 .

Tabela 1. Distribuição dos estudantes do sexo feminino de acordo com comportamento alimentar com tendência para ON e insatisfação corporal $(n=123)$

\begin{tabular}{|c|c|c|c|c|}
\hline \multirow{2}{*}{$\begin{array}{l}\text { Comportamento com tendência } \\
\text { para } 0 \mathrm{~N}\end{array}$} & \multicolumn{2}{|c|}{ Insatisfação corporal } & \multirow{2}{*}{$\frac{\text { rho }(p)}{0,25(0,001)}$} & \multirow{2}{*}{$\frac{x^{2}(p)}{7,48(0,005)}$} \\
\hline & Sim & Não & & \\
\hline Sim & 69 & 41 & & \\
\hline Não & 5 & 13 & & \\
\hline Total & 74 & 54 & & \\
\hline
\end{tabular}

\section{Ortorexia nervosa e estado nutricional}

Associação significativa também foi observada entre ON e estado nutricional ( $r h o=0,22 ; p \leq 0,05$ ), com maior frequência de estudantes com excesso de peso entre aqueles com comportamento alimentar com tendência para a ON $\left(x^{2}=\right.$ 6,56; $p=0,010$ ) (Tabela 2). Ainda, correlação negativa e significativa foi encontrada entre a pontuação do ORTO-15 e o peso corporal (rho $=-0,23 ; p=0,005)$.

A frequência de estudantes com baixo peso foi de 6,4\% $(n=09)$; desses, $88,9 \%(n=08)$ apresentaram comportamento com tendência para a ON.

Tabela 2. Distribuição dos estudantes de acordo com comportamento alimentar com tendência para ON e estado nutricional $(n=141)$

\begin{tabular}{|c|c|c|c|c|}
\hline \multirow{2}{*}{$\begin{array}{l}\text { Comportamento com tendência } \\
\text { para ON }\end{array}$} & \multicolumn{2}{|c|}{$\begin{array}{l}\text { Excesso de peso } \\
\left(I M C \geq 25 \mathrm{~kg} \cdot \mathrm{m}^{-2}\right)\end{array}$} & \multirow{2}{*}{$\begin{array}{c}\text { rho }(p) \\
-0,23(0,005)\end{array}$} & \multirow{2}{*}{$\frac{x^{2}(p)}{6,56(0,010)}$} \\
\hline & Sim & Não & & \\
\hline Sim & 34 & 89 & & \\
\hline Não & 00 & 18 & & \\
\hline Total & 34 & 107 & & \\
\hline
\end{tabular}

\section{Ortorexia nervosa e período cursado}

Não foi observada associação significativa entre o período cursado e o comportamento alimentar com tendência para $\mathrm{ON}(\mathrm{rho}=-0,03 ; \mathrm{p}=0,721)$, sem diferença para a frequência de ON encontrada no grupo de estudantes do primeiro e segundo ano vs. o grupo do terceiro e quarto ano do curso $\left(x^{2}=4,49 ; p=0,611\right)$.

Também não foi observada correlação entre a pontuação do ORTO-15 e a idade dos participantes $(r=0,032 ; p=0,703)$.

\section{Modelo explicativo para a ortorexia nervosa}

O modelo final manteve apenas as variáveis inter-relacionadas peso, altura e IMC, que explicaram a presença da ON para 13,0\% da amostra. Dada a dependência da variável IMC das demais, removeu-se tal variável do modelo, que passou a explicar 16,0\% das respostas de ON (Tabela 3). Apesar de a insatisfação corporal não ter se mantido como fator independente associado à ON neste modelo, ela apresentou forte associação com o excesso de peso (OR: 6,37, IC 95\%: $2,07-19,68, p<0,0001)$.

Tabela 3. Regressão logística explicativa para a ortorexia nervosa

\begin{tabular}{lccc}
\hline Variáveis & $\boldsymbol{\beta}$ & $\mathbf{I C 9 5 \%}$ & $\mathbf{p}$ \\
\hline Peso $(\mathrm{kg})$ & 1,12 & $1,04-1,19$ & 0,002 \\
Altura $(\mathrm{m})$ & 0,01 & $0,00-4,65$ & 0,011 \\
Constante & 962,32 & & 0,281 \\
\hline
\end{tabular}

B: beta; IC 95\%: intervalo de confiança de 95\%; p: nível de significância.

\section{DISCUSSÃO}

Nutricionistas e estudantes de nutrição parecem ser um público com maior vulnerabilidade para desenvolver ON. De fato, no presente trabalho, encontramos que a grande maioria dos estudantes de nutrição avaliados apresentou esse desvio do comportamento alimentar. Corroborando a maior vulnerabilidade para a ON nesse público, Pontes ${ }^{8}$, Souza e Rodrigues ${ }^{18}$ e Alvarenga et al..$^{19}$ também encontraram alta prevalência de comportamento com tendência a ON em estudantes do curso técnico em nutrição (83,0\%), estudantes de nutrição $(88,7 \%)$ e nutricionistas $(81,9 \%)$, respectivamente. No cenário internacional, estudos recentes confirmam essa mesma tendência entre estudantes de nutrição ${ }^{29}$ e nutricionistas ${ }^{30}$.

A maior suscetibilidade de nutricionistas e futuros nutricionistas apresentarem comportamentos de risco para ON guarda relação direta com as atividades inerentes a essa profissão, que trabalha diretamente com questões relacionadas à alimentação e ao corpo. Indo ao encontro dos nossos achados, Malmborg et al..$^{31}$ verificaram que estudantes de educação física - profissão que também lida com essas 
questões - apresentaram maior tendência de apresentar comportamentos sugestivos de ortorexia quando comparados a estudantes de negócios (84,5\% vs. 65,4\%, respectivamente, $p=0,002$ ).

Estudantes de nutrição e nutricionistas comumente apresentam preocupação excessiva com a composição nutricional e com o valor calórico dos alimentos, encarando o alimento com um olhar puramente biológico, como um mero fornecedor de nutrientes para a manutenção da saúde, e esse comportamento pode não ser muito saudável, especialmente quando consideramos o contexto da ON. Em realidade, esse fenômeno se constitui em um paradoxo no qual o comer saudável não é saudável. Pessoas com comportamento de risco para ortorexia estão, especialmente do ponto de vista psicológico, adoecidas, ainda que seu peso e IMC estejam de acordo com os padrões de normalidade estabelecidos. A comida ocupa diversos e diferentes papéis em nossa vida, e seus aspectos culturais, sociais, emocionais e políticos são de extrema importância e devem ser valorizados, principalmente pelos nutricionistas, cujo trabalhar é lidar de forma direta e íntima com os alimentos. A visão unilateral, com valorização apenas do aspecto biológico/nutricional, é uma forma reducionista e medicalizada de olhar para a comida, além de não ser benéfica para a saúde, especialmente quando entendemos saúde de forma mais ampla, tal como proposto pela $\mathrm{OMS}^{32}$.

Em nosso estudo, altas prevalências de ON foram observadas desde os períodos iniciais do curso e sem diferenças entre os anos cursados. Resultados semelhantes foram observados por Souza e Rodrigues ${ }^{18}$, também com estudantes de nutrição, e por Pontes ${ }^{8}$, com estudantes do curso técnico de nutrição. A avaliação desses resultados sugere que tais comportamentos parecem existir antes mesmo do início do curso, estando relacionados com a própria escolha da profissão. Indivíduos que já apresentam preocupações excessivas com o tema alimentação, corpo e saúde podem optar pelo curso de nutrição justamente por esse curso se destacar pelo estudo aprofundado dos alimentos, suas composições e funções no organismo, bem como das associações entre alimentação e saúde/doença ${ }^{20,33}$.

Certamente não seria prudente sugerir que todos os indivíduos que se preocupam em ter uma alimentação saudável estão em risco de desenvolver ON. Todavia, deve-se ter cautela com a aparente "inocente" preocupação em se ter uma alimentação saudável, pois isso pode sinalizar o início de desajustes na relação do indivíduo com a comida, especialmente se considerarmos o recente fenômeno da ON. Nesse caso, o que se inicia como uma simples preocupação em comer de forma saudável, pode evoluir de forma progressiva, com restrições alimentares cada vez maiores ao longo do tempo, podendo gerar a eliminação de grupos alimentares inteiros e a realização de jejuns parciais (prática comumente adotada atualmente como estratégia para perda de peso) como forma de desintoxicação do organismo. Tal preocupação patológica em apenas comer alimentos saudáveis pode gerar problemas médicos, pessoais e sociais ${ }^{9}$.

Embora a perda de peso não faça parte de objetivos primários dos indivíduos com ON ${ }^{9,34}$, ela é apontada como um possível fator desencadeador da $\mathrm{ON}^{5,6}$. A realização de restrições alimentares e a adoção de dietas consideradas "corretas" e saudáveis é uma estratégia largamente utilizada por indivíduos que almejam redução do peso e/ou alteração do formato corporal ${ }^{23}$, e isso pode servir como gatilho para o início do desenvolvimento de comportamentos alimentares associados à ON. A insatisfação com a imagem corporal e o excesso de peso estão diretamente associados a prática de dietas, e estudos anteriores já demonstraram que experiências frequentes com dietas aumentam a tendência para o desenvolvimento de $\mathrm{ON}^{17,35,36}$. A maior frequência de insatisfação corporal e de excesso de peso entre os estudantes com tendência à ON, que foi observada no presente estudo, se alinha com essa premissa. No modelo de regressão explicativo da ON, apenas as variáveis peso e altura se mostraram fatores independentemente associados à $\mathrm{ON}$, apontando a associação entre estado nutricional e ON. E, apesar de a insatisfação corporal não ter se mantido como fator associado à ON nesse modelo, ela apresentou forte associação com o excesso de peso. Oberle et al. ${ }^{37}$ verificaram que, entre os homens, valores maiores de IMC se associaram com maior sintomatologia para a ON; Barnes e Caltabiano ${ }^{38}$ encontraram associação entre baixa satisfação com a imagem corporal e maior tendência para ON. Em estudo realizado com 1.899 adolescentes poloneses, foi visto que o comportamento ortoréxico esteve relacionado a maior IMC e que os indivíduos que estavam fazendo dieta também apresentaram maior pontuação para a ON ${ }^{39}$; e entre atletas, a realização prévia de dietas foi um dos preditores independentes de $\mathrm{ON}^{40}$. Essas associações apoiam a hipótese de que o comportamento sugestivo de ON pode ser confundido, a princípio, com alternativa para se alcançar perda de peso e/ou mudança da forma corporal, especialmente pela confusão existente entre "fazer dieta" e "comer de forma saudável", que são comumente vistos como sinônimos. Por outro lado, é importante destacar que alguns trabalhos que também avaliaram associações entre ON, estado nutricional e satisfação corporal não encontraram associação entre esses fatores ${ }^{18,41}$.

O presente estudo possui algumas limitações que devem ser consideradas na interpretação dos achados. Por tratar-se de um estudo transversal, não é possível fazer inferências sobre relação de causalidade entre as variáveis. Outra fragilidade está associada ao instrumento utilizado para avaliar a ON, que apresenta algumas limitações. Os pontos de corte adotados no ORTO-15 e algumas perguntas que o compõem devem ser considerados dentro dessas limitações ${ }^{19}$, 
pois podem levar a alguns equívocos e fazer com que sejam identificados com tendência à ON indivíduos que são conscientes sobre a importância de se alimentar bem, que possuam ideologias alimentares específicas, como os veganos, ou que simplesmente estejam realizando alguma restrição alimentar por um tempo limitado ${ }^{42}$. Portanto, recomenda-se cautela na interpretação e generalização dos resultados deste e dos estudos na temática em geral, pois não seria adequado considerar que um fenômeno tão recente, complexo e pouco compreendido atinja níveis de prevalência tão altos. Estudos futuros, utilizando critérios diagnósticos mais específicos, aplicados por meio de entrevista clínica, como os propostos por Dunn e Bratman?, são importantes para que tenhamos um melhor panorama tanto da prevalência quanto dos fatores associados à ON.

Todavia, apesar de ser necessária cautela em relação às prevalências obtidas nos estudos, não se pode ignorar que esse desvio do comportamento alimentar merece aprofundamento, tanto em relação aos critérios diagnósticos quanto em relação aos fatores disparadores e associados. Nesse sentido, nosso estudo é importante, pois expande a literatura sobre a ON e contribui para ampliar os conhecimentos nesse tema tão atual e relevante, especialmente entre estudantes de nutrição.

\section{CONCLUSÃO}

Os resultados do presente estudo demonstram que os estudantes de nutrição parecem ser uma população especialmente em risco para tendência à $\mathrm{ON}$, independentemente do período cursado. A maior frequência de estudantes com ON e excesso de peso e insatisfação corporal podem sugerir que tais condições, que comumente cursam com preocupações com alimentação saudável, podem facilitar o início do desenvolvimento de comportamentos alimentares associados à ON.

Tais achados sinalizam que é fundamental a discussão dos conceitos de "alimentação saudável" e do que é "ser saudável" durante a formação profissional do nutricionista. Pelas maiores exigências e por sofrer fortes pressões sociais, inerentes à própria profissão, em relação ao peso, aparência e qualidade da alimentação, esse público é mais vulnerável para o desenvolvimento de desvios do comportamento alimentar como a ON. E essa é uma questão preocupante e que não deve ser negligenciada ao longo da formação, uma vez que crenças equivocadas sobre o que, de fato, significa comer de forma saudável, podem comprometer o processo de cuidado e aconselhamento nutricional. Se o próprio futuro nutricionista não consegue estabelecer uma relação adequada e equilibrada com o comer e com a comida, dificilmente conseguirá verdadeiramente ajudar seus pacientes e clientes a compreenderem a alimentação de forma ampla, não apenas sob uma perspectiva biológica, mas com um olhar para os importantes aspectos sociais, culturais e emocionais que a compõem. Da mesma forma, estudos de cunho qualitativo e quantitativo, que se aprofundem na investigação de fatores associados à ON, são importantes para o melhor entendimento e, consequentemente, abordagem deste fenômeno.

\section{CONTRIBUIÇÕES INDIVIDUAIS}

Maria Eduarda Araújo - Executou a revisão bibliográfica, a coleta e análise dos dados, a interpretação dos resultados e a redação do artigo.

Sabrina M. Barroso - Realizou a análise estatística dos dados, revisou criticamente e aprovou a versão final do artigo.

Camila C. Japur - Revisou criticamente a escrita e aprovou a versão final do artigo.

Fernanda R. O. Penaforte - Idealizou o trabalho e orientou a escrita e o desenvolvimento do artigo (concepção, delineamento, análise e interpretação dos dados), revisou criticamente a escrita e aprovou a versão final do artigo.

\section{CONFLITOS DE INTERESSE}

Os autores declaram não haver conflito de interesse.

\section{AGRADECIMENTOS}

Ao curso de graduação em Nutrição da Universidade Federal do Triângulo Mineiro, por disponibilizar a estrutura física necessária para a realização do presente trabalho.

\section{REFERÊNCIAS}

1. Martins MCT, Alvarenga MS, Vargas SVA, Sato KSCJ, Scagliusi FB. Ortorexia nervosa: reflexões sobre um novo conceito. Rev Nutr. 2011;24(2):345-57.

2. Bartrina JA. Ortorexia o la obsesión por la dieta saludable. Arch Latinoam Nutr. 2007;57(4):313-5.

3. Bratman S, Knight D. Health food junkies: orthorexia nervosa: overcoming the obsession with healthful eating. New York: Broadway Books; 2001.

4. Matieu J. What is ortorexia? J Am Diet Assoc. 2005;105(10):1510-2.

5. Catalina Zamora ML, Bote Bonaechea B, García Sánchez F, Ríos Rial B. [Orthorexia nervosa. A new eating behavior disorder?]. Actas Esp Psiquiatr. 2005;33(1):66-8

6. Sánchez RM, Moreno AM. Ortorexia y vigorexia: nuevos transtornos de la conducta alimentaria? Transtornos de la Conducta Alimentaria. 2007; 5:457-82.

7. Donini LM, Marsili D, Graziani MP, Imbriale M, Cannella C. Orthorexia nervosa: validation of a diagnosis questionnaire. Eat Weight Disord. 2005;10(2):e28-32. 
8. Pontes JB. Ortorexia em estudantes de nutrição: a hipercorreção incorporada ao habitus profissional? [dissertação]. Brasília: Universidade de Brasília; 2012.

9. Dunn TM, Bratman S. On orthorexia nervosa: A review of the literature and proposed diagnostic criteria. Eat Behav. 2016;21:11-7.

10. American Psychiatry Association. Diagnostic and Statistical Manual of Mental disorders DSM-5. 5th ed. Washington: American Psychiatric Association; 2013.

11. Stochel M, Janas-Kozik M, Zejda J, Hyrnik J, Jelonek I, Siwiec A. [Validation of ORTO-15 Questionnaire in the group of urban youth aged 15-21]. Psychiatr Pol. 2015;49(1):119-34.

12. Brytek-Matera A, Krupa M, Poggiogalle E, Donini LM. Adaptation of the ORTH0-15 test to Polish women and men. Eat Weight Disord. 2014;19(1):69-76.

13. Dunn TM, Gibbs J, Whitney N, Starosta A. Prevalence of orthorexia nervosa is less than $1 \%$ : data from a US sample. Eat Weight Disord. 2017;22(1):185-192.

14. Herranz Valera J, Acuña Ruiz P, Romero Valdespino B, Visioli F. Prevalence of orthorexia nervosa among ashtanga yoga practitioners: a pilot study. Eat Weight Disord. 2014;19(4):469-72.

15. Bağci Bosi AT, Camur D, Güler C. Prevalence of orthorexia nervosa in resident medical doctors in the faculty of medicine (Ankara, Turkey). Appetite. 2007;49(3):661-6.

16. Donini LM, Marsili D, Graziani MP, Imbriale M, Cannella C. Orthorexia nervosa: a preliminary study with a proposal for diagnosis and an attempt to measure the dimension of the phenomenon. Eat Weight Disord. 2004;9(2):151-7.

17. Kinzl JF, Hauer K, Traweger C, Kiefer I. Orthorexia nervosa in dieticians. Psychother Psychosom. 2006;75(6):395-6.

18. Souza QJOV, Rodrigues AM. Comportamento de risco para ortorexia nervosa em estudantes de nutrição. J Bras Psiquiatr. 2014;63(3):200-4.

19. Alvarenga MS, Martins MC, Sato KS, Vargas SV, Philippi ST, Scagliusi FB. Orthorexia nervosa behavior in a sample of Brazilian dietitians assessed by the Portuguese version of ORTO-15. Eat Weight Disord. 2012;17(1):e29-35.

20. Korinth $A, S$ Chiess $S$, Westenhoefer J. Eating behaviour and eating disorders in students of nutrition sciences. Public Health Nutr. 2010;13(1):32-7.

21. Fidan T, Ertekin V, Işikay S, Kirpinar I. Prevalence of orthorexia among medical students in Erzurum, Turkey. Compr Psychiatry. 2010;51(1):49-54.

22. Araújo KL, Pena PGL, Freitas MCS, Diez-Garcia RW. Estigma do nutricionista com obesidade no mundo do trabalho. Rev Nutr. 2015;28(6):569-79.

23. Pontes JB, Montagner MI, Montagner MA. Ortorexia nervosa: adaptação cultural do Orto15. Demetra. 2014;9(2):533-48.

24. Cordás TA, Castilho S. Imagem corporal nos transtornos alimentares - instrumento de avaliação: "Body Shape Questionnaire". Psiquiatria Biológica. 1994;2(1):17-21.

25. Branco LM, Hilário MOE, Cintra IP. Percepção e satisfação corporal em adolescentes e a relaçãa com seu estado nutricional. Rev Psiquiatr Clín. 2006;33(6):292-6.

26. Brasil. Vigilância alimentar e nutricional - Sisvan: orientações básicas para a coleta, o processamento, a análise de dados e a informação em serviços de saúde. Brasília: Ministério da Saúde; 2004.
27. World Health Organization. Obesity: preventing and managing the global epidemic. Report of a WHO Consulation. Geneva: World Health Organization; 1998 (Technical Report Series, nº 894).

28. de Onis M, Onyango AW, Borghi E, Siyam A, Nishida C, Siekmann J. Development of a WHO growth reference for school-aged children and adolescents. Bull World Health Organ. 2007;85(9):660-7.

29. Gubiec E, Stetkiewicz-Lewandowicz A, Rasmus P, Sobów T. Problem ortoreksji w grupie studentów kierunku dietetyka [Orthorexia in a group of dietetics students]. Medycyna Ogólna i Nauki o Zdrowiu. 2015;21(1):95-100.

30. Asil E, Sürücüoğlu MS. Orthorexia Nervosa in Turkish Dietitians. Ecol Food Nutr. 2015;54(4):303-13

31. Malmborg J, Bremander A, Olsson MC, Bergman S. Health status, physical activity, and orthorexia nervosa: A comparison between exercise science students and business students. Appetite. 2017;109:137-143.

32. WHO (World Health Organization). Constitution of the World Health Organization. Basic Documents. Geneve; 1946.

33. Hagen MEK, Negri ST, Ramos M. Influências na escolha por curso de nutrição em calouros de Porto Alegre (RS). Cad Educação. 2011;39:221-41.

34. Varga M, Dukay-Szabó S, Túry F, van Furth EF. Evidence and gaps in the literature on orthorexia nervosa. Eat Weight Disord. 2013;18(2):103-11.

35. Missbach B, Hinterbuchinger B, Dreiseitl V, Zellhofer S, Kurz C, König J. When Eating Right, Is Measured Wrong! A Validation and Critical Examination of the ORT0-15 Questionnaire in German. PLoS One. 2015;10(8):e0135772.

36. Varga M, Thege BK, Dukay-Szabó S, Túry F, van Furth EF. When eating healthy is not healthy: orthorexia nervosa and its measurement with the ORTO-15 in Hungary. BMC Psychiatry. 2014;14:59.

37. Oberle CD, Samaghabadi RO, Hughes EM. Orthorexia nervosa: Assessment and correlates with gender, BMI, and personality. Appetite. 2017;108:303-10.

38. Barnes MA, Caltabiano ML. The interrelationship between orthorexia nervosa, perfectionism, body image and attachment style. Eat Weight Disord. 2017;22(1):177-84.

39. Hyrnik J, Janas-Kozik M, Stochel M, Jelonek I, Siwiec A, Rybakowski JK. The assessment of orthorexia nervosa among 1899 Polish adolescents using the ORT0-15 questionnaire. Int J Psychiatry Clin Pract. 2016;20(3):199-203.

40. Segura-García C, Papaianni MC, Caglioti F, Procopio L, Nisticò CG, Bombardiere L, et al. Orthorexia nervosa: a frequent eating disordered behavior in athletes. Eat Weight Disord. 2012;17(4):e226-33.

41. Sanlier N, Yassibas E, Bilici S, Sahin G, Celik B. Does the rise in eating disorders lead to increasing risk of orthorexia nervosa? Correlations with gender, education, and body mass index. Ecol Food Nutr. 2016;55(3):266-78.

42. Håman L, Barker-Ruchti N, Patriksson G, Lindgren EC. Orthorexia nervosa: an integrative literature review of a lifestyle syndrome. Int I Qual Stud Health Well-being. 2015;10:26799. 